\title{
Size-effects on the surface tension near the critical point : Monte Carlo simulations of the Lennard-Jones fluid.
}

\author{
Florent Goujon ${ }^{\mathrm{a}}$, Aziz Ghoufib ${ }^{\mathrm{b}}$, Patrice Malfreyt ${ }^{\mathrm{a}, *}$ \\ ${ }^{a}$ Université Clermont Auvergne, CNRS, SIGMA Clermont, Institut de Chimie de Clermont-Ferrand (ICCF), F-63000 Clermont-Ferrand, France. \\ ${ }^{b}$ Institut Physique de Rennes, UMR CNRS 6251, Université Rennes 1, 35042 Rennes, France
}

\begin{abstract}
We report Monte Carlo (MC) simulations of the Lennard-Jones (LJ) fluid at the liquid-vapor interface in the critical region. A slab-based tail method is associated with the MC simulations to approach as close as possible the critical point $\left(T^{*}=0.98 T_{C}^{*}\right)$. We investigate then the impact of system-sizes on the surface tension and coexisting densities by considering very large box dimensions for which the surface tension is independent of system-sizes at low temperatures.
\end{abstract}

Keywords: Surface tension, critical point, Monte Carlo simulations, slab-based tail method

\section{Introduction}

Most of the phenomena in surface science adhesion, wetting, lubrication [1, 2], nucleation [3, 4] involve the combination of liquid-liquid, liquid-vapor, and liquid-solid interfaces. An understanding and control of the surface tension is at the heart of many important industrial and practical processes. The molecular simulations have been extensively used this last decade [5] to reproduce the interfacial tension at different thermodynamic conditions and to describe the interface region in terms of specific arrangements $[6,2]$.

Since 1974 and so far, the calculation of the surface tension from two-phase simulations has been an area of active research. This long-standing activity is explained by the fact that a number of factors such as the surface area (See Figures 1a and 1b) $[7,8,9,10,11,12]$, the cutoff radius and the corresponding long-range corrections $[13,14,15,16,17,18,19,20]$, the truncation of the potential and the force as well as the method used for the calculation of the surface tension $[21,22,23,24,25,26,27]$ make the simulated properties dependent on initial conditions. All these issues have been addressed in a recent review [5]. A number of recommendations have been proposed to resolve these issues leading now to an accurate calculation of the surface tension for reduced temperatures less than $0.85 T_{C}^{*}$.

For higher temperatures, the situation is far from being identical. Indeed, the cutoff-dependence on the surface tension and coexisting densities is still stronger [28]. Recently, we have shown that it is possible to approach the gas-liquid critical point of the Lennard-Jones fluid by performing two-phase simulations of a slab geometry. These simulations have used a slab-based tail [17] methodology with large cutoff values. The main conclusions of this work are that Monte Carlo simulations with the addition of long-range corrections to the energy during the course of the

*Corresponding author: Patrice.Malfreyt@uca.fr 
simulations using a reduced cutoff of $r_{c}^{*}=6.4$ reproduce quantitatively the dependencies of the densities and surface tensions on the temperature close to the critical point. In the case of the modeling of methane, the real value of the cutoff corresponds to $r_{c}=6.4 \sigma=24 \AA$ which is twice that used in most atomistic simulations [6, 26].

Whereas a reduced cutoff of $r_{c}^{*}=3.0$ is large enough to avoid any cutoff-dependence of the surface tension with a slab-based tail method for temperatures far from the critical point (see Ref.12), an increase to $r_{c}^{*}=6.4$ is required to provide reliable surface tensions and coexisting densities in the critical region [28]. A key question arises now : for this large cutoff value of $r_{c}^{*}=6.4$, how do the size-effects affect the values of surface tension and coexisting densities of the critical region? The size-effects will be evaluated trough the variations of the interfacial area $A=L_{x} \times L_{y}$ and the longitudinal dimension $L_{z}$ where $z$ represents the direction normal to the interface. It has been established, at relatively low temperatures, that the size-effects led to oscillatory behaviors of the surface tension with the surface area (Figure 1a) and the $\mathrm{L}_{z}^{*}$ dimension (Figure $1 \mathrm{~b}$ ).
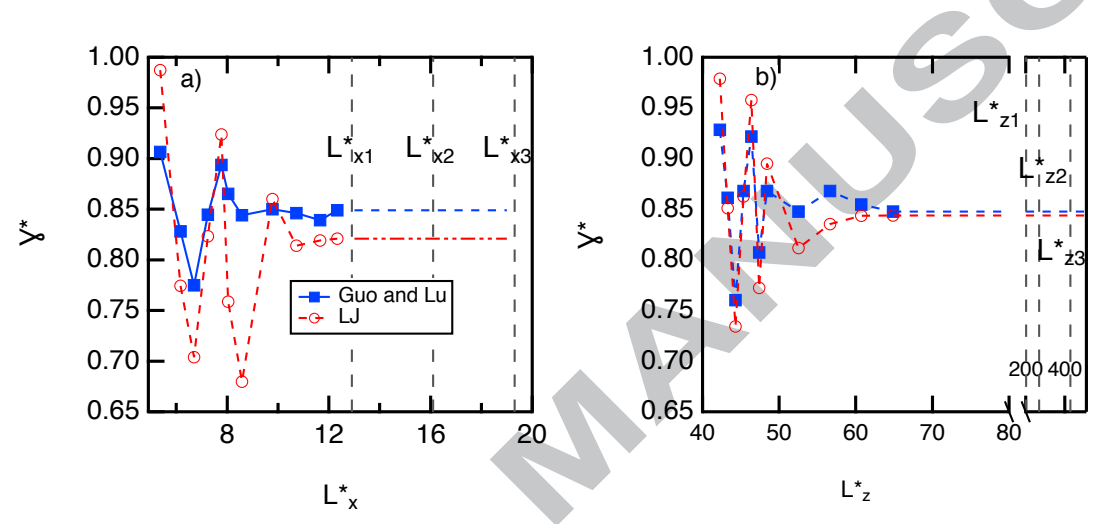

Figure 1. Surface tensions [10] calculated with the truncated LJ potential and the LJ potential modified with the Guo and Lu slab-based tail methodology at $T^{*}=0.8$ for a) different interfacial areas and b) $L_{z}^{*}$ dimensions. The dotted vertical lines represent the values of interfacial area and of longitudinal dimensions $L_{z}^{*}$ investigated here.

To address this issue, we propose here to investigate LJ liquid-vapor systems close to the critical point with large surface areas and longitudinal dimensions. Figures $1 \mathrm{a}$ and $1 \mathrm{~b}$ show the values of surface areas $\left(L_{x 1}^{*}, L_{x 2}^{*}, L_{x 3}^{*}\right)$ and longitudinal dimensions $\left(L_{z 1}^{*}, L_{z 2}^{*}, L_{z 3}^{*}\right)$ considered here. The details of the simulation in terms of box sizes and number of LJ particles are given in Table 1 .

\section{Methodology}

All the properties reported here refer to the Lennard-Jones $(\mathrm{LJ})$ particle and are expressed in reduced LJ units where $T^{*}=k_{B} T / \epsilon, \gamma^{*}=\gamma \sigma^{2} / \epsilon, L^{*}=L / \sigma, p^{*}=p \sigma^{3} / \epsilon, \rho^{*}=\rho \sigma^{3}$ where $T^{*}, \gamma^{*}, L^{*}, p^{*}, \rho^{*}$ represent the reduced temperature, surface tension, length, pressure and density, respectively. MC simulations were performed in the constantNVT ensemble. All simulations were organized in cycles. Each cycle consisted of $N$ translations. The equilibration phase was composed of 500000 cycles and the production phase of $2 \times 10^{6}$ cycles up to $5 \times 10^{10}$ translation moves. The maximum displacement which was adjusted during the equilibrium phase to give an acceptance ratio of 0.4 , was found to be close to 0.23 at $T^{*}=1.26$ and to 0.26 at $T^{*}=1.28$ in reduced LJ units. The thermodynamic and mechanical properties were calculated every 20 cycles requiring the storage of 100000 configurations. The statistical errors for these properties were estimated using 5 superblocks averages of 20000 configurations. The MC calculations were carried out at two reduced temperatures $T^{*}=1.26$ and 1.28 for a cutoff radius $r_{\mathrm{c}}^{*}=6.4$.

In the Janeček approach, the long-range correction to the truncated potential is added to the truncated $u_{\mathrm{ST}}\left(r_{i j}\right)$ potential. Considering a system of $N$ atoms, the total configurational energy $U_{\text {TOт }}$ is

$$
U_{\mathrm{TOT}}=\sum_{i=1}^{N-1} \sum_{j=i+1}^{N} u_{\mathrm{ST}}\left(r_{i j}\right)+U_{\mathrm{LRC}}
$$


where $r_{i j}$ is the pair separation distance and $u_{\mathrm{ST}}\left(r_{i j}\right)$ is the spherically-truncated Lennard-Jones potential defined by

$$
u_{\mathrm{ST}}\left(r_{i j}\right)= \begin{cases}u_{\mathrm{LJ}}\left(r_{i j}\right) & r_{i j}<r_{\mathrm{c}} \\ 0 & r_{i j} \geq r_{\mathrm{c}} .\end{cases}
$$

$u_{\mathrm{LJ}}$ is the Lennard-Jones (LJ) potential defined by $\epsilon$ and $\sigma$ corresponding to energy and size parameters, respectively. The simulation box is divided into $N_{s}$ slabs of width $\Delta z=0.13$ (in LJ units). Each slab, which is parallel to the interface, has a volume $V_{s}=L_{x} L_{y} \Delta z$ and $z_{k}$ defines the centre of the $k$ th slab. In Equation (1), $U_{\text {LRC }}$ is defined as

$$
U_{\mathrm{LRC}}=\frac{1}{2} \sum_{k=1}^{N_{s}} u_{\mathrm{lrc}}\left(z_{k}\right)
$$

where the long-range correction energy of the slab $k$ is

$$
u_{\operatorname{lrc}}\left(z_{k}\right)=\rho\left(z_{k}\right) V_{s} \sum_{j=1}^{N_{s}} \rho\left(z_{j}\right) w\left(\left|z_{j}-z_{k}\right|\right) \Delta z
$$

where the sum is over all the slabs in the box. $\rho\left(z_{k}\right)$ defines the density number of the slab $k$. The contribution $w(\xi)=w\left(\left|z_{j}-z_{k}\right|\right)$ is calculated by assuming a uniform distribution of atoms in the slab. For the Lennard-Jones potential, the function $w(\xi)$ is

$$
w(\xi)= \begin{cases}4 \pi \epsilon \sigma^{2}\left[\frac{1}{5}\left(\frac{\sigma}{r_{c}}\right)^{10}-\frac{1}{2}\left(\frac{\sigma}{r_{c}}\right)^{4}\right] & \text { for } \xi \leq r_{c} \\ 4 \pi \epsilon \sigma^{2}\left[\frac{1}{5}\left(\frac{\sigma}{\xi}\right)^{10}-\frac{1}{2}\left(\frac{\sigma}{\xi}\right)^{4}\right] & \text { for } \xi>r_{c}\end{cases}
$$

Note that Equation (3) defines the long-range correction energy as a sum over slabs whereas the original paper [17] considers the correction in terms of a molecule $i$ at $z_{i}$. Finally there is an improvement to the original version of Janeček's method proposed by MacDowell and Blas [29], which avoids the decomposition of the long-range correction of the energy into local contributions and increases the speed of the calculation. The long-range corrections to the energy are applied at each MC move although the density profile used to calculated the long-range corrections is updated every $10 \mathrm{MC}$ cycles [12].

The intrinsic part of the surface tension, $\gamma_{\mathrm{I}}$, calculated using the Irving and Kirkwood [21, 22, 30, 31, 32, 33] definition, uses the components $p_{N}(z)$ and $p_{T}(z)$ of the pressure tensor as a function of $z$

$$
\gamma_{\mathrm{I}}=\int_{-L_{z} / 2}^{L_{z} / 2}\left(p_{\mathrm{N}}(z)-p_{\mathrm{T}}(z)\right) d z .
$$

Equation (6) is a mechanical definition of $\gamma$ based upon the force acting across a unit area in the $z$-plane for one interface. Due to the truncation of the potential, the long range corrections to the surface tension are calculated with the Janeček approach as

$$
\gamma_{\text {Irc }}\left(z_{k}\right)=\frac{V_{s}}{A}\left[p_{\mathrm{N}, \operatorname{lrc}}\left(z_{k}\right)-p_{\mathrm{T}, \operatorname{lrc}}\left(z_{k}\right)\right]
$$

The normal and tangential components of the long-range corrections of the pressure tensor are given by

$$
p_{\mathrm{N}, \operatorname{lrc}}\left(z_{k}\right)=\rho\left(z_{k}\right) \sum_{j=1}^{N_{s}} \rho\left(z_{j}\right) \pi_{z z}\left(\left|z_{j}-z_{k}\right|\right) \Delta z
$$


and

$$
\begin{aligned}
p_{\mathrm{T}, \mathrm{Irc}}\left(z_{k}\right) & =\frac{1}{2}\left(p_{x x, \operatorname{lrc}}\left(z_{k}\right)+p_{y y, \operatorname{lrc}}\left(z_{k}\right)\right) \\
& =\rho\left(z_{k}\right) \sum_{j=1}^{N_{s}} \rho\left(z_{j}\right) \frac{1}{2}\left(\pi_{x x}\left(\left|z_{j}-z_{k}\right|\right)\right. \\
& \left.+\pi_{y y}\left(\left|z_{j}-z_{k}\right|\right)\right) \Delta z
\end{aligned}
$$

where the operational expressions of the functions $\pi_{x x}(\xi), \pi_{y y}(\xi)$ and $\pi_{z z}(\xi)$ can be found in Ref.12.

Table 1. Reduced surface tensions of the $\mathrm{LJ}$ fluid calculated at different surface areas $A$ and $\mathrm{L}_{z}^{*}$ dimensions where $N$ represents the total number of particles in the box. The different contributions of the surface tension, i.e, intrinsic, long range and total parts are given in LJ reduced units. $<\gamma>$

\begin{tabular}{|c|c|c|c|c|c|c|}
\hline $\mathrm{L}_{x}^{*} \times \mathrm{L}_{y}^{*}$ & $\mathrm{~L}_{z}^{*}$ & $N$ & Box & $\gamma_{\mathrm{I}}^{*}$ & $\gamma_{\text {LRC }}^{*}$ & $\gamma^{*}$ \\
\hline & & & $\mathrm{T}^{*}=1.2$ & & & \\
\hline $12.9 \times 12.9$ & 200.9 & 10796 & $\mathrm{~A}_{1} \mathrm{~L}_{z 1}$ & $0.055_{10}$ & $0.008_{1}$ & $0.063_{10}$ \\
\hline $16.1 \times 16.1$ & 200.9 & 16856 & $\mathrm{~A}_{2} \mathrm{~L}_{z 1}$ & $0.065_{7}$ & $0.012_{1}$ & $0.077_{7}$ \\
\hline $19.3 \times 19.3$ & 200.9 & 24259 & $\mathrm{~A}_{3} \mathrm{~L}_{z 1}$ & $0.063_{7}$ & $0.012_{1}$ & $0.075_{7}$ \\
\hline $12.9 \times 12.9$ & 267.9 & 16195 & $\mathrm{~A}_{1} \mathrm{~L}_{z 2}$ & $0.083_{20}$ & $0.013_{1}$ & $0.096_{20}$ \\
\hline $12.9 \times 12.9$ & 428.6 & 26992 & $\mathrm{~A}_{1} \mathrm{~L}_{z 3}$ & $0.089_{37}$ & $0.015_{1}$ & $0.104_{37}$ \\
\hline $12.9 \times 12.9$ & 200.9 & 10796 & $\mathrm{~A}_{1} \mathrm{~L}_{z 1}$ & $0.027_{10}$ & $0.005_{1}$ & $0.032_{10}$ \\
\hline $16.1 \times 16.1$ & 200.9 & 16856 & $\mathrm{~A}_{2} \mathrm{~L}_{z 1}$ & $0.038_{12}$ & $0.010_{1}$ & $0.048_{12}$ \\
\hline $19.3 \times 19.3$ & 200.9 & & $\mathrm{~A}_{3} \mathrm{~L}_{z 1}$ & $0.038_{3}$ & $0.006_{1}$ & $0.045_{3}$ \\
\hline $12.9 \times 12.9$ & 267.9 & 16195 & $\mathrm{~A}_{1} \mathrm{~L}_{z 2}$ & $0.044_{13}$ & $0.008_{1}$ & $0.052_{13}$ \\
\hline $12.9 \times 12.9$ & 428.6 & 26992 & $\mathrm{~A}_{1} \mathrm{~L}_{z 3}$ & $0.061_{13}$ & $0.009_{1}$ & $0.070_{13}$ \\
\hline
\end{tabular}
is calculated using the Irving-Kirkwood definition. The subscripts give the accuracy of the last decimal(s), i.e., $0.104_{37}$ means $0.104 \pm 0.037$. The surface area $A_{1}$ corresponds to $12.9 \times 12.9$ and the $\mathrm{L}_{z 1}$ dimension to 200.9 in reduced units.

\section{Results and discussions}

Figure 2 shows the profiles of the difference between the normal and tangential pressure components at $\mathrm{T}^{*}=1.28$ for both surface areas. First, we observe that the profiles of $p_{\mathrm{N}}^{*}\left(z^{*}\right)-p_{\mathrm{T}}^{*}\left(z^{*}\right)$ and $\gamma^{*}\left(z^{*}\right)$ are in line with an expected behavior of liquid-vapor interface at mechanical equilibrium. However, the peaks at the interface are much less marked at $\mathrm{T}^{*}=1.28$. The profiles of $\gamma^{*}\left(z^{*}\right)$ shows that the bulk phases do not provide any contribution to the surface tension and lead to flat portions on the profiles. Only the interface contributes to this property indicating that it is stable and well-located. In other words, it is possible to simulate explicit interfaces of LJ fluid very close to the critical point $\left(0.98 T_{C}^{*}\right) . T_{C}^{*}$ was estimated to 1.31 by GCMC-FSC simulations [34]. We also observe that a larger system-size $\left(A_{3} L_{z 1}\right)$ leads to a different surface tension. The difference of surface tension between these two surface areas comes from the contribution of the interfaces, with peaks of slightly larger magnitudes for $A_{1} L_{z 1}$. The fluctuations in the bulk phases are identical between the two systems-sizes. As result, the investigation of the impact of the system-sizes on the surface tension is relevant in the critical region at these two temperatures.

We now focus on the heart of this work, namely the effects of the system-sizes close to the critical point. Figure 3 and Table 1 show the dependencies of the surface tension on the interfacial area $\left(L_{x}^{*}=\sqrt{(A)}\right)$ and on the longitudinal dimension $\left(L_{z}^{*}\right)$ at both temperatures $T^{*}=1.26$ and $T^{*}=1.28$. We also report the impact of the box dimensions on the liquid and vapor densities. First, we check that the surface tensions and coexisting densities respect the temperature dependence even if the variation of temperature $\left(\Delta T^{*}=0.02\right)$ is weak. The decreases of the surface tension and liquid density and the increase of the vapor density are always observed despite the system-size. Second, we are able 


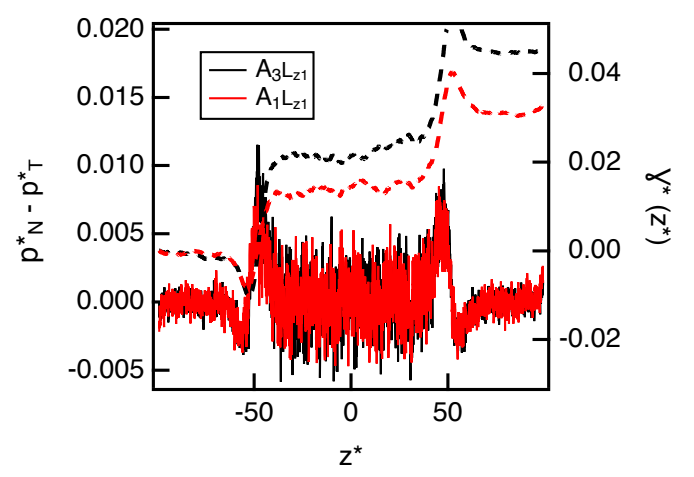

Figure 2. Profiles of the difference $p_{\mathrm{N}}^{*}\left(z^{*}\right)-p_{\mathrm{T}}^{*}\left(z^{*}\right)$ along the direction $z^{*}$ normal to the interface at $\mathrm{T}^{*}=1.28$. The integral of these profiles are represented by dotted lines on the right axis.

to provide very small values of surface tensions (smaller than 0.027 in reduced units) with a slab-based tail method. The long range correction to the surface tension contributes to about $15 \%$ of the total value. This tail contribution can never be neglected even at high temperatures. With intensive MC calculations, we obtain very small values of fluctuations in the surface tension making the comparison of the interfacial properties with different system-sizes relevant.
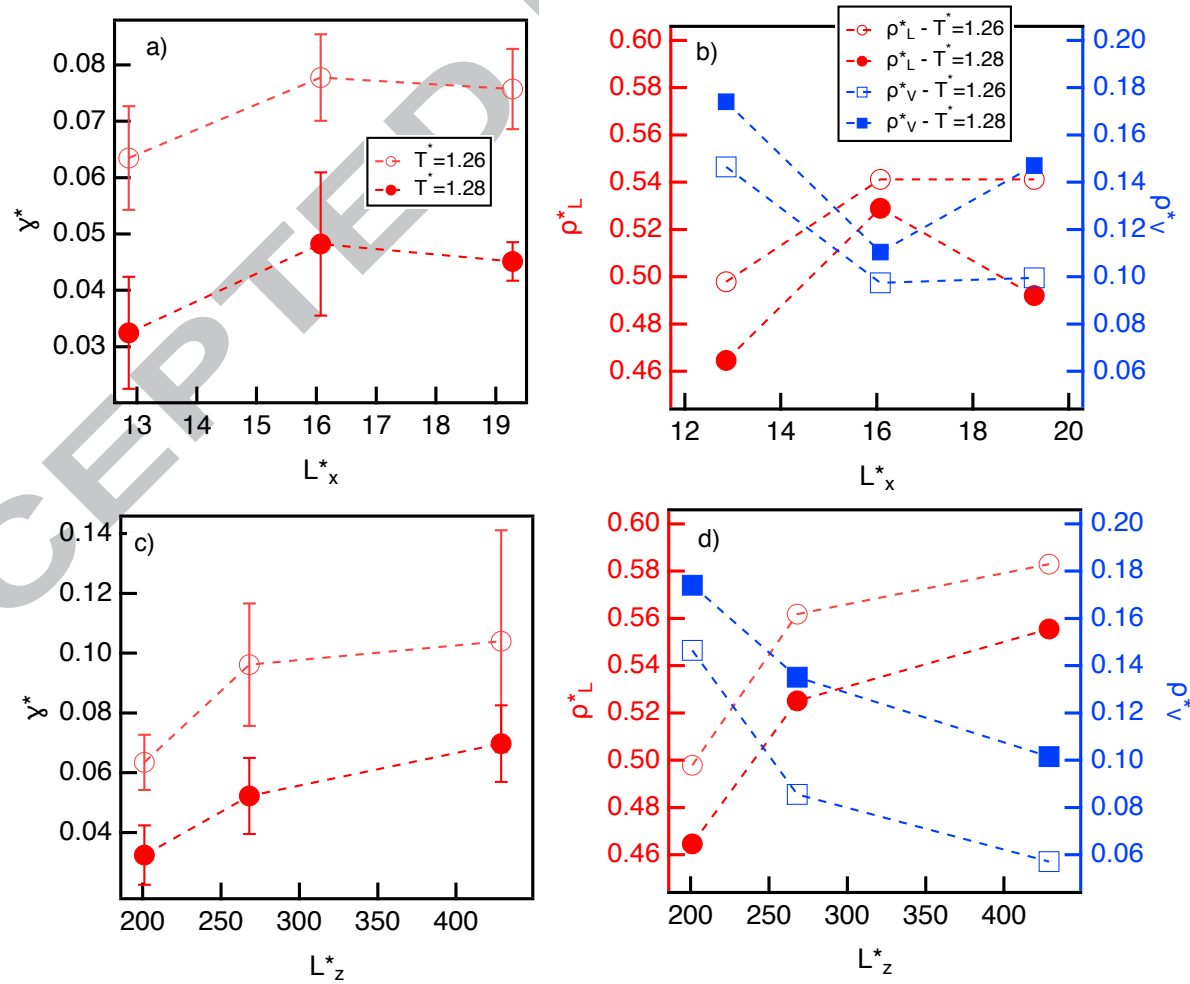

Figure 3. a) and c) Reduced surface tensions and b) and d) coexisting densities as a function of the interfacial area $\left.\left(L_{x}^{*}=\sqrt{(} A\right)\right)$ and the longitudinal dimension $L_{z}^{*}$ at two reduced temperatures $T^{*}=1.26$ and 1.28. In parts b) and d), the liquid and vapor densities are represented by red points and blue points on the right and left axes, respectively. 
Third, for lower temperatures $\left(T^{*}<0.85 T_{C}^{*}\right)$, the maximum variation of the surface tension in the first oscillations of $\gamma^{*}=f\left(L_{x}^{*}\right)$ reaches $30 \%$ with the LJ truncated potential and decreases to $15 \%$ with the slab-based tail methodology used here (see Figure 1). When the surface tension does not change any more with $L_{x}^{*}$ (from $L_{x}^{*}>11$ ), the values of $\gamma$ are identical within $0.8 \%$. As shown in part b) of Figure 1, these same variations in $\gamma^{*}=f\left(L_{z}^{*}\right)$ are about $20 \%$ with the Janeček method and the surface tension is independent on $L_{z}^{*}$ from $L_{z}^{*}=60$. The dimensions of the simulation boxes simulated here (see Figure 1 and Table 1) are significantly larger than those recommended to avoid any size-effects at smaller temperatures. Nevertheless, the size-effects are still noticeable for these very large box dimensions close to the critical point. Actually, parts a) and c) of Figure 3 show variations of surface tension in the order of $35 \%$ with the interfacial area (Figure 3a) and the longitudinal dimension (Figure 3c). It means that it is not possible to reach a surface tension independent on the system-size close to the critical region within the range of system-sizes investigated here. As expected as we approach $T_{C}$, the size-effects become more important. We can draw the same conclusions with the coexisting densities. The liquid density supports variations in the order of $20 \%$ whereas the vapor density changes by $45 \%$ with $L_{x}^{*}$ and $L_{z}^{*}$. This is an interesting result since it has been shown $[35,7]$ in the past that the coexisting densities were not dependent on system-sizes for temperatures lower than $0.90 T_{C}^{*}$.

The density profiles of Figure 4 confirm the dependencies of the coexisting densities on system-sizes. For the same interfacial area $A_{1}$, the density profiles show a clear dependence on $L_{z}^{*}$. The density profiles can be fitted to a hyperbolic tangent function even if the agreement is less satisfactory in the interfacial region. The density profiles in the bulk regions are less flat than at lower temperatures and the interface is more diffuse. This is the price to be paid when we approach the critical point. In addition, a weak variation $\left(\Delta T^{*}=0.2\right)$ of temperature is clearly observable on the profiles. Anyway, these profiles are rather well defined to allow an estimate of the coexisting densities. These density profiles show that the interfacial thickness increases significantly as we approach $T_{C}$. It is well-known that the interfacial width coincides with the bulk correlation length $[36,37,38]$ and thus diverges at $T_{C}^{*}$ as $d^{*} \sim\left(T_{\mathrm{C}}^{*}-T^{*}\right)^{\nu}$. We have shown in a recent paper [28] that this divergence of the interfacial thickness starts at $T^{*}=1.28$ and that the slope $v$ obtained by Monte Carlo simulations was equal to $-0.52 \pm 0.02$ whereas the mean-field theory [30] predicts $v=-1 / 2$. In Figure 4b, we have represented the interfacial thickness $d^{*}$ as a function of $T_{C}^{*}-T^{*}$. The values were taken from a recent work [28] for which the dimensions of the system match are a bit smaller than those of $A_{1} L_{z 1}$. The values $d^{*}$ calculated here are reported on this figure for different box dimensions. We observe a good agreement with the value of $d^{*}$ calculated in $A_{1} L_{z 1}$ and strong deviations when the longitudinal box dimension $L_{z}^{*}$ is changing. Actually, the strong dependence of the interfacial thickness on $L_{z}^{*}$, illustrated in part a) of this figure, is confirmed on this curve. We cannot conclude on the scaling exponent since additional simulations must be carried out with the box dimensions used in this paper. To complete this comparison, we have represented in Figure 4c the surface tension $\gamma^{*}$ as a function of $T_{C}^{*}-T^{*}$. Our MC simulations [28] predict a slope $\mu$ of $1.12 \pm 0.0 .2$ in $\gamma^{*} \sim\left(T_{\mathrm{C}}^{*}-T^{*}\right)$ whereas $\mu=1.26$ in the mean-field theory [30]. We observe here that changing $L_{z}$ impacts strongly on the values of $\gamma^{*}$ when approaching $T_{C}^{*}$.

\section{Summary}

The combination of a slab-based tail method with Monte Carlo simulations has been used to investigate the dependencies of the coexistence properties close to the critical point at $T^{*}=0.98 T_{C}^{*}$. It has been established at low temperatures $\left(T^{*}<0.85 T_{C}^{*}\right)$ that the surface tension shows an oscillatory behavior with the interfacial area for interfacial areas smaller than $\left(L_{x}^{*} \times L_{y}^{*}<11 \times 11\right)$. In this work, we took the route of using large box dimensions for investigating the critical region. The main conclusion is that the surface tension and the coexisting densities are system-sizes dependent even with very large box dimensions. The surface tension is dependent on both the interfacial area and the longitudinal dimension. The variation of the surface tension as a function of $L_{x}^{*}$ or $L_{z}^{*} \operatorname{can}$ reach $35 \%$. The liquid and vapor density significantly change with the box dimensions. None of these dependencies can be attributed to large statistical fluctuations and unstable interfaces. All these factors have been controlled by doing intensive Monte Carlo simulations with a slab-based tail method. We also report a strong dependence of the surface tension and the interfacial thickness on the longitudinal dimension when approaching $T_{C}^{*}$. 

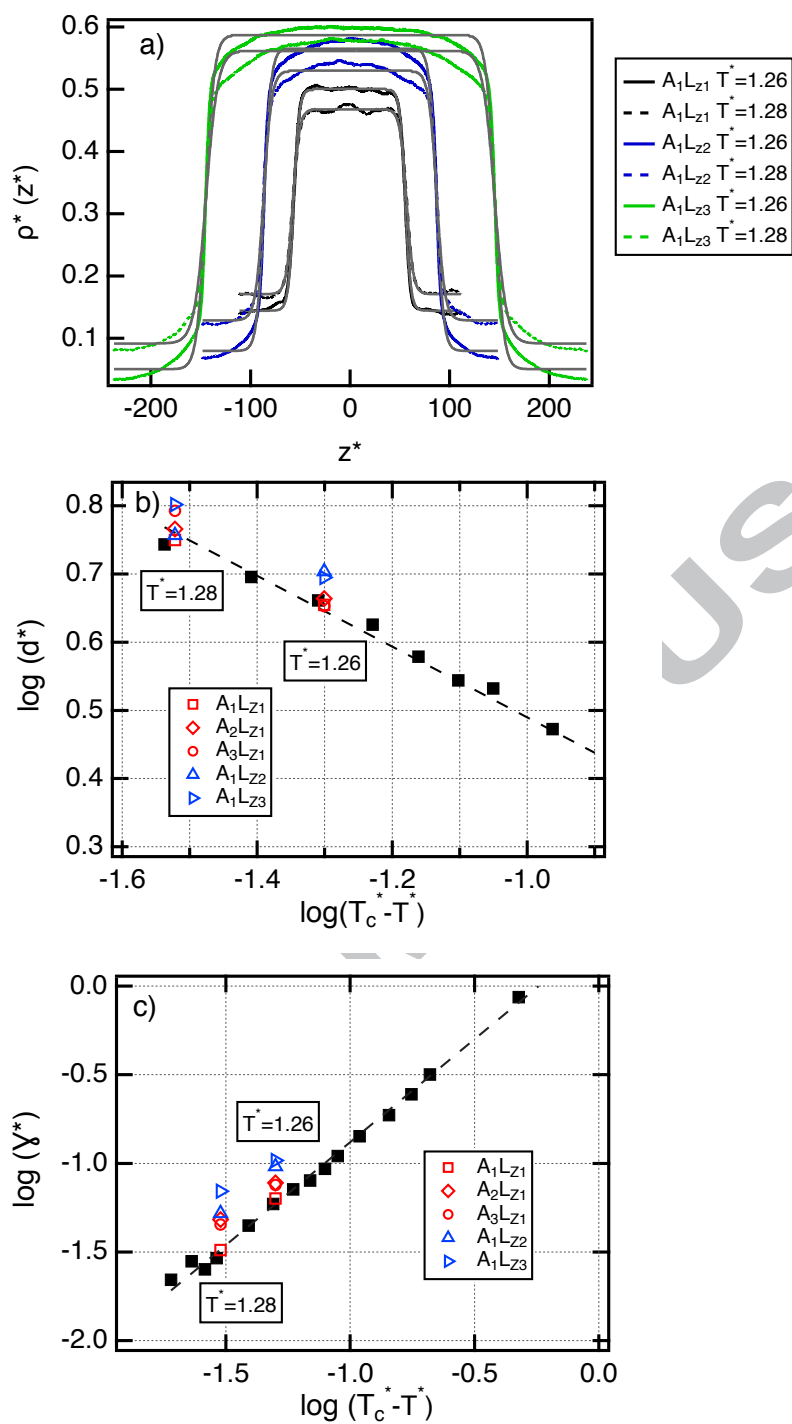

Figure 4. a) Density profiles of the LJ fluid at $T^{*}=1.26$ and $T^{*}=1.28$ for different system-sizes as indicated in Table 1 . We add for discussion the fits of these density profiles to a hyperbolic tangent function [28]; Log-log plots of the b) interfacial width $d^{*}$ and c) reduced surface tension $\gamma^{*}$ versus $T_{C}^{*}-T^{*}$ where $T_{C}^{*}$ was taken to 1.31 [34]. The dotted lines represent the least-square fits [28]. The interfacial widths and surface tensions calculated in this work are represented for different box dimensions as indicated in the legend.

\section{References}

[1] A. C. Mendonca, P. Malfreyt, A. A. Padua, Interactions and ordering of ionic liquids at a metal surface, J. Chem. Theory. Comput. 8 (2012) 3348-3355.

[2] A. C. Mendonca, A. A. Padua, P. Malfreyt, Nonequilibrium molecular simulations of new ionic lubricants at metallic surfaces: Prediction of the friction, J. Chem. Theory. Comput. 9 (2013) 1600-1610.

[3] S. Auer, D. Frenkel, Suppression of crystal nucleation in polydisperse colloids due to increase of the surface free energy, Nature 413 (2001) 711-713.

[4] S. Prestipino, A. Laio, E. Tosatti, Systematic improvement of classical nucleation theory, Phys. Rev. Lett. 108 (2012) 225701.

[5] A. Ghoufi, P. Malfreyt, D. J. Tildesley, Computer modelling of the surface tension of the gas-liquid and liquid-liquid interface, Chem. Soc. Rev. 45 (2016) 1387-1409. 
[6] N. Ferrando, V. Lachet, J. Pérez-Pellitero, A. D. Mackie, P. Malfreyt, A. Boutin, A transferable force field to predict phase equilibria and surface tension of ethers and glycol ethers., J. Phys. Chem. B 115 (2011) 10654-10664.

[7] P. Orea, J. Lopez-Lemus, J. Alejandre, Oscillatory surface tension due to finite-size effects, J. Chem. Phys. 123 (2005) 114702.

[8] M. Gonzalez-Melchor, P. Orea, J. Lopez-Lemus, F. Bresme, J. Alejandre, Stress anisotropy induced by periodic boundary conditions, J. Chem. Phys. 122 (2005) 094503.

[9] J. R. Errington, D. A. Kofke, Calculation of surface tension via area sampling, J. Chem. Phys. 127 (2007) 174709

[10] F. Biscay, A. Ghoufi, F. Goujon, V. Lachet, P. Malfreyt, Calculation of the surface tension from monte carlo simulations: Does the model impact on the finite-size effects?, J. Chem. Phys. 130 (2009) 184710.

[11] S. Werth, S. V. Lishchuk, M. Horsch, H. Hasse, The inflence of the liquid slab thickness on the planar vapor-liquid interfacial tension, Physica A 392 (2013) 2359-2367.

[12] F. Goujon, A. Ghoufi, P. Malfreyt, D. J. Tildesley, Controlling the long-range corrections in atomistic monte carlo simulations of two-phase systems, J. Chem. Theory Comput. 11 (2015) 4575-4585.

[13] E. Salomons, M. Mareschal, Atomistic simulation of liquid-vapour coexistence:binary mixtures, J. Phys. Condens. Matter 3 (1991) 92159228.

[14] E. M. Blokhuis, D. Bedaux, C. D. Holcomb, J. A. Zollweg, Tail corrections to the surface tension of a Lennard-Jones liquid-vapour interface, Molec. Phys. 85 (1995) 665-669.

[15] M. Guo, B. Lu, Long range corrections to thermodynamic properties of inhomogeneous systems with planar interfaces, J. Chem. Phys. 106 (1997) 3688-3695.

[16] F. Goujon, P. Malfreyt, A. Boutin, A. H. Fuchs, Direct monte carlo simulations of the equilibrium properties of n-pentane liquid-vapor interface, J. Chem. Phys. 116 (2002) 8106-8117.

[17] J. Janeček, Long range corrections in inhomogeneous simulations, J. Chem. Phys. 131 (2006) 6264-6269.

[18] C. Ibergay, A. Ghoufi, F. Goujon, P. Ungerer, A. Boutin, B. Rousseau, P. Malfreyt, Molecular simulations of the n-alkane liquid-vapor interface: Interfacial properties and their long range corrections, Phys. Rev. E 75 (2007) 051602.

[19] V. K. Shen, R. D. Mountain, J. R. Errington, Comparative study of the effect of tail corrections on surface tension determined by molecular simulation, J. Phys. Chem. B 111 (2007) 6198-6207.

[20] J. M. Míguez, M. M. Piñeiro, F. J. Blas, Influence of the long-range corrections on the interfacial properties of molecular models using Monte Carlo simulation, J. Chem. Phys. 138 (2013) 34707-34716.

[21] J. G. Kirkwood, F. P. Buff, The statistical mechanical theory of surface tension, J. Chem. Phys. 17 (1949) $338-343$.

[22] J. H. Irving, J. Kirkwood, The statistical mechanical theory of transport processes .IV. The equations of hydrodynamics, J. Chem. Phys. 18 (1950) 817-829.

[23] F. P. Buff, Some considerations of surface tension, Zeit. Elecktrochem. 56 (1952) 311-313.

[24] F. P. Buff, Spherical interface. ii. molecular theory, J. Chem. Phys. 23 (1955) 419-427.

[25] G. J. Gloor, G. Jackson, F. J. Blas, E. de Miguel, Test-area simulation method for the direct determination of the interfacial tension of systems with continuous or discontinuous potentials, J. Chem. Phys. 123 (2005) 134703-134721.

[26] A. Ghoufi, F. Goujon, V. Lachet, P. Malfreyt, Multiple histogram reweighting method for the surface tension calculation, J. Chem. Phys. 128 (2008) 154718 .

[27] B. B. Laird, R. L. Davidchack, Y. Yang, M. Asta, Determination of the solid-liquid interfacial free energy along a coexistence line by gibbs-cahn integration, J. Chem. Phys. 131 (2009) 114110.

[28] F. Goujon, A. Ghoufi, P. Malfreyt, D. J. Tildesley, Can we approach the gas-liquid critical point using slab simulations of two coexisting phases ?, J. Chem Phys. 145 (2016) 124702.

[29] L. G. MacDowell, F. Blas, Surface tension of fully flexible Lennard-Jones chains: role of long-range corrections, J. Chem. Phys. 131 (2009) $074705-074714$.

[30] J. S. Rowlinson, B. Widom, Molecular Theory of Capillarity, Clarendon Press, Oxford, 1982.

[31] J. P. R. B. Walton, D. J. Tildesley, J. S. Rowlinson, J. R. Henderson, The pressure tensor at the planar surface of a liquid, Molec. Phys. 48 (6) (1983) 1357-1368.

[32] J. P. R. B. Walton, D. J. Tildesley, J. S. Rowlinson, J. R. Henderson, The pressure tensor at the planar surface of a liquid, Molec. Phys. 50 (6) (1983) 1381, erratum.

[33] F. J. Martinez-Ruiz, F. J. Blas, B. Mendiboure, A. I. M.-V. Bravo, Effect of dispersive long-range corrections to the pressure tensor. the vapour-liquid interfacial properties of the lennard-jones system revisited., J. Chem. Phys. 141 (2014) 184701.

[34] J. J. Potoff, A. Panagiotopoulos, Surface tension of the three-dimensional Lennard-Jones fluid from histogram reweighting Monte Carlo simualtions, J. Chem. Phys. 112 (2000) 6411-6415.

[35] L. J. Chen, Area dependence of the surface-tension of a Lennard-Jones fluid from molecular-dynamics simulations, J. Chem. Phys. 103 (23) (1995) 10214-10216.

[36] F. H. Stillinger, Capillary waves and the inherent density profile for the liquid-vapor interface, J. Chem Phys. 75 (1982) 1087-1091.

[37] V. Russier, J.-M. Caillo, Statistical field theory for liquid vapor interface, Condens. Matter Phys. 13 (2) (2010) 23602.

[38] E. Mayoral, A. Gama Goicochea, Hyperscaling relationship between the interfacial tension of liquids and their correlation length near the critical point, Soft Matter 10 (2014) 9054-9058. 
- We investigate the size-effects on the surface tension in the critical region

- We approach the critical as close as possible with a slab-based tail Monte Carlo method.

- The surface tension and coexisting densities are system-sizes dependent in the critical region. 
